\title{
A vanishing theorem and symbolic powers of planar point ideals
}

\author{
M. Dumnicki, T. Szemberg $\dagger$ and H. Tutaj-Gasińska
}

\begin{abstract}
The purpose of this paper is twofold. We present first a vanishing theorem for families of linear series with base ideal being a fat points ideal. We then apply this result in order to give a partial proof of a conjecture raised by Bocci, Harbourne and Huneke concerning containment relations between ordinary and symbolic powers of planar point ideals.
\end{abstract}

\section{Introduction}

One of the central problems in the theory of linear series is the study of linear systems of hypersurfaces in projective spaces with assigned base schemes. This problem is related to various other topics, for example to the polynomial interpolation, the Waring problem, the classification of defective higher secant varieties to mention a few in the realms of the algebraic geometry, to the problem of containment relations between ordinary and symbolic powers of ideals in commutative algebra and to problems in combinatorics [8].

Given a finite number $s$ of points $P_{1}, \ldots, P_{s}$ in a projective space $\mathbb{P}^{n}$ and fixed integers $m_{1}, \ldots, m_{s}$, one is interested in determining the dimension of the linear system $\mathcal{L}=$ $\mathcal{L}_{n}\left(t ; m_{1}, \ldots, m_{s}\right)$ of hypersurfaces of a fixed degree $t$ vanishing in the given set of points with prescribed multiplicities. The virtual dimension of this space

$$
\operatorname{vdim}(\mathcal{L})=\left(\begin{array}{c}
n+t \\
n
\end{array}\right)-\sum_{i=1}^{s}\left(\begin{array}{c}
n+m_{i}-1 \\
n
\end{array}\right)-1
$$

arises by assuming that the conditions imposed by the underlying set of points are independent. The expected dimension

$$
\operatorname{edim}(\mathcal{L})=\max \{\operatorname{vdim}(\mathcal{L}),-1\}
$$

is just a modification of $\operatorname{vdim}(\mathcal{L})$ taking into account the convention that the empty set has dimension -1 . One always has

$$
\operatorname{dim}(\mathcal{L}) \geqslant \operatorname{edim}(\mathcal{L})
$$

If there is the equality in (1), then we say that the linear system $\mathcal{L}$ is non-special. Otherwise the system is special.

A subscheme $Z$ of $\mathbb{P}^{n}$ defined by an ideal of the form

$$
\mathcal{I}_{Z}=\mathfrak{m}_{P_{1}}^{m_{1}} \cap \ldots \cap \mathfrak{m}_{P_{s}}^{m_{s}},
$$

where $\mathfrak{m}_{P}$ denotes the maximal ideal of a point $P \in \mathbb{P}^{n}$ is called a fat points scheme and the ideal $\mathcal{I}_{Z}$ is called a fat points ideal. It follows from the long cohomology sequence attached to the twisted structure sequence of $Z$

$$
0 \rightarrow \mathcal{I}_{Z}(t) \rightarrow \mathcal{O}_{\mathbb{P}^{n}}(t) \rightarrow \mathcal{O}_{Z}(t) \rightarrow 0
$$

Received 6 February 2013; revised 18 June 2013.

2010 Mathematics Subject Classification 14C20 (primary), 14F17, 14H50, 14J26, 13A15, $13 \mathrm{~F} 20$ (secondary).

${ }^{\dagger}$ The second named author was partially supported by NCN grant UMO-2011/01/B/ST1/04875. 
that the system $\mathcal{L}$ is non-special exactly when the cohomology group

$$
H^{1}\left(\mathbb{P}^{n}, \mathcal{I}_{Z}(t)\right)
$$

vanishes. The system $\mathcal{L}$ is called $h^{1}$-regular if it is non-special and effective, see Definition 3.1.

In the case of the projective plane, the non-speciality of linear series of type $\mathcal{L}$ is governed by the beautiful geometrical Segre-Harbourne-Gimigliano-Hirschowitz (SHGH) conjecture, see for example $[\mathbf{1}, \S 4]$ for a precise statement and historical background.

Since the SHGH conjecture seems out of reach at present, it is of interest to provide other criteria ensuring the vanishing of the cohomology group in (3). Our first main result is the following vanishing theorem.

Theorem A. Let $P_{1}, \ldots, P_{s} \in \mathbb{P}^{2}$ be $s \geqslant 4$ general planar points. Let $m_{1} \geqslant m_{2} \geqslant \ldots \geqslant$ $m_{s} \geqslant 1$ be fixed integers. If

$$
t \geqslant m_{1}+m_{2} \quad \text { and } \operatorname{vdim}\left(\mathcal{L}_{2}\left(t ; m_{1}, \ldots, m_{s}\right)\right) \geqslant \frac{1}{2}\left(3 m_{4}^{2}-7 m_{4}+2\right),
$$

then

$$
h^{1}\left(\mathbb{P}^{2}, \mathcal{O}_{\mathbb{P}^{2}}(t) \otimes \mathfrak{m}_{P_{1}}^{m_{1}} \otimes \ldots \otimes \mathfrak{m}_{P_{s}}^{m_{s}}\right)=0
$$

that is the system $\mathcal{L}_{2}\left(t ; m_{1}, \ldots, m_{s}\right)$ is non-special.

Turning to the algebraic side of the story, let $\mathcal{I} \subset \mathbb{C}\left[\mathbb{P}^{n}\right]=\mathbb{C}\left[x_{0}, \ldots, x_{n}\right]$ be a homogeneous ideal. The $m$ th symbolic power $\mathcal{I}^{(m)}$ of $\mathcal{I}$ is defined as

$$
\mathcal{I}^{(m)}=\mathbb{C}\left[\mathbb{P}^{n}\right] \cap\left(\bigcap_{\mathfrak{p} \in \operatorname{Ass}(\mathcal{I})} \mathcal{I}^{m} \mathbb{C}\left[\mathbb{P}^{n}\right]_{\mathfrak{p}}\right),
$$

where the intersection is taken in the field of fractions of $\mathbb{C}\left[\mathbb{P}^{n}\right]$. If $\mathcal{I}$ is a fat points ideal as in (2), then the symbolic power is simply given by

$$
\mathcal{I}^{(m)}=\bigcap_{i=1}^{s} \mathfrak{m}_{P_{i}}^{m \cdot m_{i}} .
$$

There has been considerable interest in containment relations between usual and symbolic powers of homogeneous ideals over the last two decades. The most general results in this direction have been obtained with multiplier ideal techniques in characteristic zero by Ein et al. [4] and using tight closures in positive characteristic by Hochster and Huneke [7]. Applying these results to a homogeneous ideal $\mathcal{I}$ in the coordinate ring $\mathbb{C}\left[\mathbb{P}^{n}\right]$ of the projective space we obtain the following containment statement

$$
\mathcal{I}^{(n r)} \subset \mathcal{I}^{r} \text { for all } r \geqslant 0 .
$$

There are examples showing that one cannot improve the power of the ideal $\mathcal{I}$ on the right-hand side of (4). Nevertheless, it is natural to wonder to what extent this result can be improved; for example, under additional geometrical assumptions on the zero-locus of $\mathcal{I}$. In particular, if $\mathcal{I}$ is a fat points ideal, it is natural to wonder for which non-negative integers $m, r$ and $j$ there is the containment

$$
\mathcal{I}^{(m)} \subset \mathcal{M}^{j} \mathcal{I}^{r}
$$

where $\mathcal{M}$ denotes the irrelevant ideal. Harbourne and Huneke suggested the following answer to that problem [6, Conjecture 2.1]. This assertion has guided our research leading to this note.

Conjecture. Let $\mathcal{I}$ be a fat points ideal in $\mathbb{P}^{n}$. Then

$$
\mathcal{I}^{(n r)} \subset \mathcal{M}^{r(n-1)} \mathcal{I}^{r}
$$

for all $r \geqslant 1$. 
This conjecture has been proved recently by Harbourne and Huneke for general points in $\mathbb{P}^{2}$ (see [6, Proposition 3.10]) and by the first author for general points in $\mathbb{P}^{3}$ (see [2, Theorem 3]). In this note we extend these results to a large family of fat points ideals in $\mathbb{P}^{2}$. More specifically we show the following theorem.

Theorem B. Let $\mathcal{I}=\mathfrak{m}_{P_{1}}^{m_{1}} \cap \ldots \cap \mathfrak{m}_{P_{s}}^{m_{s}}$ be a fat points ideal supported on $s \geqslant 9$ general points in $\mathbb{P}^{2}$. If one of the following conditions holds:

(a) at least $s-1$ among the $m_{i}$ are equal (almost homogeneous case);

(b) $m_{1} \geqslant \ldots \geqslant m_{s} \geqslant m_{1} / 2$ (uniformly fat case);

then the conjecture above holds, that is, there is the containment

$$
\mathcal{I}^{(2 r)} \subset \mathcal{M}^{r} \cdot \mathcal{I}^{r}
$$

for all $r \geqslant 1$.

We hope that more technical statements in Theorems 3.2 and 4.4 could be of independent interest when dealing with problems of similar flavor to those studied in this note.

\section{A reduction procedure}

In this section we are concerned with some operations on finite sequences of integers (which later on will be sequences of multiplicities). We begin by fixing some notation.

Definition 2.1. Let $S=\left(a_{1}, a_{2}, \ldots, a_{k}\right)$ be a sequence of non-negative integers. We call the number

$$
\operatorname{size}(S)=\sum_{i=1}^{k} a_{i}
$$

the size of the sequence $S$.

We say that a sequence $\left(a_{1}, \ldots, a_{k}\right)$ is dominated by a sequence $\left(b_{1}, \ldots, b_{\ell}\right)$ if

$$
k \leqslant \ell \text { and } a_{i} \leqslant b_{i} \text { for all } i=1, \ldots, k .
$$

The following reduction process was introduced in $[\mathbf{3}]$.

\section{REDUCTION ALGORITHM WITH PARAMETER $m$}

Let $S=\left(b_{1}, \ldots, b_{k}, a_{1}, \ldots, a_{m}\right)$ be a sequence of positive integers.

$Z_{m}:=\{1,2, \ldots, m\}$

for all $k=m, m-1, \ldots, 1$ do

$$
\begin{aligned}
& z_{k}:=\max \left(Z_{k}\right) \\
& \text { if }\left(a_{k}<m \text { and } a_{k} \leqslant z_{k}\right) \text { then } r_{k}:=a_{k} \text { else } r_{k}:=z_{k} \mathbf{f i}
\end{aligned}
$$$$
c_{k}:=a_{k}-r_{k}
$$$$
Z_{k-1}:=Z_{k} \backslash\left\{r_{k}\right\}
$$

od$$
\text { if } Z_{k-1}=Z_{k} \text { then stop fi }
$$

If the above process terminates by stop, then we say that the sequence $S$ is not $m$-reducible. Otherwise $S$ is $m$-reducible. The new sequence

$$
\left(b_{1}, \ldots, b_{k}, c_{1}, \ldots, c_{m}\right)
$$

is in that case called the $m$-reduction of $S$. Note that

$$
\operatorname{size}\left(b_{1}, \ldots, b_{k}, c_{1}, \ldots, c_{m}\right)=\operatorname{size}\left(b_{1}, \ldots, b_{k}, a_{1}, \ldots, a_{m}\right)-\frac{m(m+1)}{2} .
$$

We call the numbers $r_{k}$ appearing in the algorithm the reducers. Thus, at each stage of the reduction algorithm $Z_{k}$ is the set of available reducers. 
The following examples explain how the algorithm works.

EXAMPLE 2.2. In the following table we present the input ( $m$ and the indispensable sequence $\left.a_{1}, \ldots, a_{m}\right)$, the reducers $r_{1}, \ldots, r_{m}$ and the resulting sequence $c_{1}, \ldots, c_{m}$, or the word stop, if the $m$-reduction fails. Algorithm steps are omitted.

$\begin{array}{ccccc} & m & a_{1}, \ldots, a_{m} & r_{1}, \ldots, r_{m} & c_{1}, \ldots, c_{m} \\ \text { (A) } & 3 & 5,5,5 & 1,2,3 & 4,3,2 \\ \text { (B) } & 4 & 5,5,3,1 & 2,4,3,1 & 3,1,0,0 \\ \text { (C) } & 3 & 4,1,3 & 2,1,3 & 2,0,0 \\ \text { (D) } & 3 & 4,2,2 & -, 2,2 & \operatorname{stop}\left(Z_{1}=Z_{2}\right) .\end{array}$

The next example is more involved. We trace the algorithm steps.

EXAMPLE 2.3. In this example we will perform a sequence of reductions in such a way that the result of $j$ th reduction will be the input for $(j+1)$ th reduction. Observe that the reduction algorithm works on a sequence of positive integers, so we must shorten the sequence of integers by deleting zeroes at the end. We will start with $\left(a_{1}, \ldots, a_{10}\right)=(1,2, \ldots, 10)$ and use $m_{j^{-}}$ reductions, $m_{1}=m_{2}=m_{3}=4, m_{4}=\ldots=m_{7}=3$. We will gather all intermediate steps in the table, together with the used reducers. The table shows what happens to each sequence member. For example the seventh element, $a_{7}=7$, has been altered to 6 , then to $5,4,1$ and finally to 0 .

\begin{tabular}{cccccccccc|c}
$a_{1}$ & $a_{2}$ & $a_{3}$ & $a_{4}$ & $a_{5}$ & $a_{6}$ & $a_{7}$ & $a_{8}$ & $a_{9}$ & $a_{10}$ & $m_{j}$ \\
1 & 2 & 3 & 4 & 5 & 6 & 7 & 8 & 9 & 10 & \\
& & & & & & -1 & -2 & -3 & -4 & 4 \\
1 & 2 & 3 & 4 & 5 & 6 & 6 & 6 & 6 & 6 & \\
& & & & & & -1 & -2 & -3 & -4 & 4 \\
1 & 2 & 3 & 4 & 5 & 6 & 5 & 4 & 3 & 2 & \\
& & & & & & -1 & -4 & -3 & -2 & 4 \\
1 & 2 & 3 & 4 & 5 & 6 & 4 & 0 & 0 & 0 & \\
& & & & -1 & -2 & -3 & & & 3 \\
1 & 2 & 3 & 4 & 4 & 4 & 1 & & & \\
& & & & -2 & -3 & -1 & & & 3 \\
1 & 2 & 3 & 4 & 2 & 1 & 0 & & & \\
& & & -3 & -2 & -1 & & & & 3 \\
1 & 2 & 3 & 1 & 0 & 0 & & & & \\
& -2 & -3 & -1 & & & & & & \\
1 & 0 & 0 & 0 & & & & & &
\end{tabular}

This example is continued in Remark 3.5

The reader may check his understanding of the reduction process verifying the claims in the following two examples.

EXAmple 2.4. The sequence $(2,3,4,5,2)$ is 4-reducible, with its 4-reduction equal to $(2,2,1,1)$.

EXAmple 2.5. The sequence $(1,2,3,3)$ is not 4-reducible (we get $r_{4}=r_{3}=3$ ).

The sequence $(3,2,4,2)$ is not 4 -reducible (we get $r_{2}=r_{4}=2$ ).

The following lemma gives first insights into what happens to a sequence of integers under the reduction algorithm. 
LEMmA 2.6. Let $S=\left(b_{1}, \ldots, b_{\ell-1}, b_{\ell}, \ldots, b_{r}\right)$ be a given sequence of integers to which the reduction algorithm has been successfully applied. Assume that the original sequence $S$ has been altered on positions with labels $\ell-1$ and $\ell$ and that the resulting sequence has the form $\left(\ldots, a_{\ell-1}, a_{\ell}, \ldots\right)$. Then either $a_{\ell}=0$ or

$$
b_{\ell-1}-b_{\ell}<a_{\ell-1}-a_{\ell}
$$

Proof. In the reduction process either $b_{\ell}$ is reduced to zero, or the number $r_{\ell}$, which we subtract from $b_{\ell}$, is chosen as a maximum $z_{\ell}$ of $Z_{\ell}$, which gives $z_{\ell-1}<z_{\ell}$. Since always $r_{\ell-1} \leqslant z_{\ell-1}$, the number $r_{\ell}$ which we subtract from $b_{\ell}$ is always greater than the number $r_{\ell-1}$ which we subtract from $b_{\ell-1}$. Thus,

$$
a_{\ell-1}-a_{\ell}=b_{\ell-1}-r_{\ell-1}-b_{\ell}+r_{\ell}=b_{\ell-1}-b_{\ell}+r_{\ell}-r_{\ell-1}>b_{\ell-1}-b_{\ell} .
$$

Corollary 2.7. Assume that $\left(a_{1}, \ldots, a_{r}\right)$ is a sequence of integers obtained by a sequence of successful reductions applied consecutively to the sequence $(1,2, \ldots, r)$ (some of $a_{j}$ might be zero). Then, for every $k=1, \ldots, r$, either $a_{k}=k$ or $a_{k} \geqslant a_{k+1} \geqslant \ldots \geqslant a_{r}$. In particular, if $a_{k}=0$ then $a_{k+1}=0$.

If, moreover, the kth position of the sequence has been altered by at least two reductions, then either $a_{k-1}>a_{k}$ or $a_{k-1}=0$.

Proof. Let us assume that $a_{k}<k$. It follows that the element on the $k$ th position has been altered in the course of at least one reduction. Since reductions work from the right to the left, also each element on the $\ell$ th position, for $\ell>k$, has been altered. Therefore, it is enough to prove that if $a_{k}$ has been altered by a reduction, then $a_{k} \geqslant a_{k+1}$. This follows immediately from Lemma 2.6. That the final property holds is straightforward.

The next lemma describes numerical properties of sequences which are not $m$-reducible. It is going to be important in the proof of Theorem 3.3.

LEMma 2.8 (A consequence of non- $m$-reducibility). Let $\left(b_{1}, \ldots, b_{r}\right)$ be a sequence of integers which is not m-reducible. Then one of the following two conditions is satisfied:

(1) $r<m$ (the sequence is too short); or

(2) there exist $k, \ell$ such that $r-m+1 \leqslant k<\ell \leqslant r$ and $b_{k} \leqslant b_{\ell}, b_{k}<m$ (the sequence has a too flat tail).

Proof. The first property is obvious, so we assume that $r \geqslant m$. We adjust the notation to match the reduction algorithm and write

$$
\left(b_{1}, \ldots, b_{r}\right)=\left(b_{1}, \ldots, b_{r-m}, a_{1}, \ldots, a_{m}\right)
$$

with $a_{i}=b_{r-m+i}$ for $i=1, \ldots, m$. Let $r_{k^{\prime}+1}, \ldots, r_{m}$ be the sequence of reducers and let $c_{k^{\prime}+1}, \ldots, c_{m}$ be the sequence resulting from the reduction algorithm right before the moment it stopped (that is, we assume that it stopped for $k^{\prime}$ ). The algorithm stops if $Z_{k^{\prime}-1}=Z_{k^{\prime}}$. This means that $r_{k^{\prime}}$ was not an element of $Z_{k^{\prime}}$, so that in particular this reducer has been used in a previous step, say with index $\ell^{\prime}>k^{\prime}$. Moreover, this implies that $r_{k^{\prime}} \neq z_{k^{\prime}}$, which going back one line in the algorithm implies that $r_{k^{\prime}}=a_{k^{\prime}}$ and consequently $a_{k^{\prime}}<m$. Thus, the claim follows with $k=r-m+k^{\prime}$ and $\ell=r-m+\ell^{\prime}$.

\section{A vanishing theorem}

We begin by recalling from $[3]$ that on $\mathbb{P}^{n}$ one can consider slightly more general linear series than of the form $\mathcal{L}_{n}\left(t ; m_{1}, \ldots, m_{s}\right)$. Specifically, let $\mathcal{L}_{G, n}\left(m_{1}, \ldots, m_{s}\right)$ be the vector space 
spanned by all monomials in the ideal generated by monomials with exponents in a fixed set $G \subset \mathbb{N}^{n}$. We suppress the index $n$ if the dimension of the ambient space is understood. Thus, for example, for $n=2$ we have

$$
\mathcal{L}\left(t ; m_{1}, \ldots, m_{s}\right)=\mathcal{L}_{D}\left(m_{1}, \ldots, m_{s}\right),
$$

where $D=\left\{(x, y) \in \mathbb{N}^{2}: x+y \leqslant t\right\}$. We extend the notions of non-speciality and $h^{1}$-regularity to linear systems of that kind, see [3, Definition 5].

Definition 3.1 (Non-speciality and $h^{1}$-regularity). We say that the linear system $\mathcal{L}_{G}\left(m_{1}, \ldots, m_{s}\right)$ is non-special if its dimension agrees with the virtual dimension.

We say that the system $\mathcal{L}_{G}\left(m_{1}, \ldots, m_{s}\right)$ is $h^{1}$-regular, if it is non-special and effective.

Theorem 3.2. Fix positive integers $d, m_{1}, \ldots, m_{s}$ and let $S_{1}=(1,2, \ldots, d, d+1)$. Assume inductively that $S_{j}$ is $m_{j}$-reducible with the reduction equal to $S_{j+1}, j=1, \ldots, s$. If $\operatorname{size}\left(S_{s+1}\right)>0$ is positive, then the linear system $\mathcal{L}\left(d ; m_{1}, \ldots, m_{s}\right)$ is $h^{1}$-regular, assuming that the multiplicities are imposed in general points.

Proof. With notation recalled above, the result follows from [3]. By [3, Definition 11] we can write $D=S_{1}$. Since $S_{j}$ is $m_{j}$-reducible to $S_{j+1}$, by [3, Corollary 19] we know that if $\mathcal{L}_{S_{j+1}}\left(m_{j+1}, \ldots, m_{s}\right)$ is non-special, then $\mathcal{L}_{S_{j}}\left(m_{j}, m_{j+1}, \ldots, m_{s}\right)$ is also non-special. We start with the trivial case of $\mathcal{L}_{S_{s+1}}($ ) (with no multiplicities imposed), which is trivially nonspecial, and go back inductively to obtain non-speciality of $\mathcal{L}_{S_{1}}\left(m_{1}, \ldots, m_{s}\right)$. Observe that non-emptiness of $S_{s+1}$ gives non-emptiness of $\mathcal{L}_{S_{1}}\left(m_{1}, \ldots, m_{s}\right)$.

Although powerful when addressing very concrete questions, the above theorem is not easily applicable under general assumptions. We present below its modification which is better suited to the proof of Theorem A.

Theorem 3.3. Let $s, d, m_{1}, \ldots, m_{s}$ be positive integers, with $s \geqslant 4$. Assume that the multiplicities are ordered $m_{1} \geqslant m_{2} \geqslant m_{3} \geqslant \ldots \geqslant m_{s}$. If $d \geqslant m_{1}+m_{2}$ and

$$
\left(\begin{array}{c}
d+2 \\
2
\end{array}\right)-\sum_{j=1}^{s}\left(\begin{array}{c}
m_{j}+1 \\
2
\end{array}\right) \geqslant\left(2 m_{4}-1\right)\left(m_{4}-1\right)+1-\frac{m_{4}\left(m_{4}+1\right)}{2}=\frac{3}{2} m_{4}^{2}-\frac{7}{2} m_{4}+2,
$$

then $\mathcal{L}\left(d ; m_{1}, \ldots, m_{s}\right)$ is non-special and $h^{1}$-regular whenever the left-hand side is nonnegative, in particular for $m_{4} \geqslant 2$.

Proof. The key idea of the proof is to apply the reduction algorithm $s$ times with $m$ equal to

$$
m_{1}, m_{2}, m_{3}, m_{5}, \ldots, m_{s}, m_{4} .
$$

We begin by showing that the first three reductions are always possible. Indeed, the sequence $(1,2, \ldots, d+1)$ is $m_{1}$-reducible since $m_{1} \leqslant d$. After $m_{1}$-reduction we obtain the sequence $\left(1,2, \ldots, d+1-m_{1}, d+1-m_{1}, d+1-m_{1}, \ldots, d+1-m_{1}\right)$ in which the terms $d+1-m_{1}$ appear $m_{1}+1$ times.

The $m_{2}$-reduction works for this sequence because we have inequalities $m_{2} \leqslant m_{1}$ and $d \geqslant m_{1}+m_{2}$. We obtain the sequence

$$
\left(1,2, \ldots, d+1-m_{1}, \ldots, d+3-m_{1}-m_{2}, d+2-m_{1}-m_{2}, d+1-m_{1}-m_{2}\right)
$$

where $d+1-m_{1}$ appears at least once.

Again, since $d \geqslant m_{1}+m_{2}$, the last element in the reduced sequence (7) is at least 1 . Since $m_{3} \leqslant m_{2}$ the $m_{3}$-reduction is possible on the sequence (7). Indeed, no situation in Lemma 2.8 
can occur. After this reduction step, some elements in the sequence could be 0 and they are removed from the tail.

If $m_{4}=1$, then also $m_{5}=\ldots=m_{s}=1$ and we are done because the Reduction Algorithm always works for multiplicity 1 . So we may assume from now on that $m_{4} \geqslant 2$.

Let us assume that for some $j>3$ the $m_{j}$-reduction failed (and that all previous reductions were possible). Let $\left(a_{1}, \ldots, a_{r}\right)$ denote the state of the sequence before the $m_{j}$-reduction. By Lemma 2.8 we have two possibilities.

Case 1 . The sequence $\left(a_{1}, \ldots, a_{r}\right)$ is too short to be reduced, that is $r<m_{j} \leqslant m_{4}$. Thus, the sequence $\left(a_{1}, \ldots, a_{r}\right)$ is dominated by $\left(1,2, \ldots, m_{4}-1\right)$ with $\operatorname{size}\left(1,2, \ldots, m_{4}-1\right)=$ $\left(m_{4}-1\right) m_{4} / 2$. A contradiction.

Case 2. Assume that the sequence is $\left(a_{1}, \ldots, a_{k}, \ldots, a_{\ell}, \ldots, a_{r}\right)$, with

$$
a_{k} \leqslant a_{\ell}, \quad a_{k}<m_{j} \text { and } r-m_{j}+1 \leqslant k .
$$

We have now again two possibilities.

Subcase 2.1. Assume that $a_{k}=k$. Then $k=a_{k}<m_{j} \leqslant m_{4}$, and taking into account (8) we have $r \leqslant 2 m_{4}-2$. Thus, the sequence $\left(a_{1}, \ldots, a_{r}\right)$ is dominated by $\left(1, \ldots, 2 m_{4}-2\right)$ and

$$
\operatorname{size}\left(1,2, \ldots, 2 m_{4}-2\right)=\left(2 m_{4}-1\right)\left(m_{4}-1\right) .
$$

A contradiction.

Subcase 2.2. Assume that $a_{k}<k$. This is the most tricky situation. By Corollary 2.7 $a_{k} \geqslant a_{k+1} \geqslant a_{k+2} \geqslant \ldots \geqslant a_{\ell} \geqslant \ldots \geqslant a_{r}$. Since $a_{k} \leqslant a_{\ell}$, we have $a_{k}=a_{k+1}=\ldots=a_{\ell}$.

Assume for a moment that

$$
\ell \geqslant 2 m_{4}
$$

We know that the $\ell$ th entry had been reduced at least once. But if it had been reduced twice or more, Corollary 2.7 would imply that $a_{\ell-1}>a_{\ell}$, which is impossible. So it was reduced exactly once.

Now we claim the following.

Claim 1. The $\ell$ th entry was not reduced by the first three reductions.

Indeed, after the $m_{1}$-reduction it would become $a_{\ell}=d+1-m_{1}$. But, we have $a_{\ell}<m_{j} \leqslant m_{2}$, which contradicts our assumption that $d \geqslant m_{1}+m_{2}$.

Reductions with $m_{2}$ and $m_{3}$ are also excluded because these reductions work only on elements reduced in the previous step (that is, either by the $m_{1}$-reduction or by the $m_{2}$-reduction). But then $a_{\ell}$ would be reduced at least twice. This contradiction justifies Claim 1.

Thus, the $\ell$ th entry was reduced by some $m_{i}$-reduction with $i>3$. Then the assumption (10) gives $a_{\ell} \geqslant 2 m_{4}-m_{j} \geqslant m_{4}$. This is a contradiction again, as the assumptions of Case 2 imply that $m_{4}>a_{\ell}$.

In this way we have proved that $\ell \leqslant 2 m_{4}-1$. Hence, $k-1 \leqslant 2 m_{4}-3$ and our sequence is dominated by $\left(1,2, \ldots, 2 m_{4}-3, m_{4}-1, \ldots, m_{4}-1\right)$ (with $m_{4}-1$ appearing at most $m_{4}$ times). In fact, it appears only twice, this is our next claim.

Claim 2. In the sequence $\left(1,2, \ldots, 2 m_{4}-3, m_{4}-1, \ldots, m_{4}-1\right)$, the $m_{4}-1$ term appears at most twice.

Assume that $a_{r} \neq 0$ (otherwise we operate on a shorter sequence). It follows that $a_{j} \neq 0$ for $j=1, \ldots, r$. The sequence $\left(a_{1}, \ldots, a_{k}, \ldots, a_{\ell}, \ldots, a_{r}\right)$ results from a $m_{j-1}$-reduction of the sequence $\left(b_{1}, \ldots, b_{k}, \ldots, b_{\ell}, \ldots, b_{r}, b_{r+1}, \ldots\right)$ for some $j \geqslant 6$.

We know that the $\ell$ th element was reduced exactly once (otherwise we would have $a_{k}>a_{\ell}$ ), hence $b_{\ell}=\ell$. Let $\tilde{k}:=r-\ell$. Observe that $b_{r}$ has been reduced to a non-zero $a_{r}$. This is possible only when the reducer for $b_{r}$ has been chosen as a maximum of non-used reducers. Hence, 
either $m_{j-1}$, as a maximal reducer, has been chosen before, or it is chosen in the $\left(m_{j-1}\right)$ reduction to reduce $b_{r}$. It follows that the maximal reducer for $b_{r-1}$ is $m_{j-1}-1$. Inductively, the maximal reducer for $b_{\ell}=b_{r-\tilde{k}}$ is $m_{j-1}-\tilde{k}$, but this implies that $b_{\ell} \leqslant a_{\ell}+\left(m_{j-1}-\tilde{k}\right)$, so we have the following sequence of inequalities:

$$
\ell=b_{\ell} \leqslant a_{\ell}+m_{j-1}-\tilde{k} \leqslant m_{4}-1+m_{4}-\tilde{k}=2 m_{4}-1+\ell-r,
$$

which gives $r \leqslant 2 m_{4}-1$. This proves Claim 2 .

That claim, together with the previous bound for $\left(a_{1}, \ldots, a_{r}\right)$, gives that $\left(a_{1}, \ldots, a_{r}\right)$ is dominated by $\left(1,2, \ldots, 2 m_{4}-3, m_{4}-1, m_{4}-1\right)$ whose size is $\left(2 m_{4}-1\right)\left(m_{4}-1\right)$.

Concluding, we see that if the size of some sequence obtained during reducing is at least $\left(2 m_{4}-1\right)\left(m_{4}-1\right)+1$, then it is $p$-reducible for each $p \leqslant m_{4}$.

The size of our sequence $(1, \ldots, d+1)$, at the beginning, is $(d+1)(d+2) / 2$. After the $j$ th reduction it is (remember that we reduce using $m_{1}, m_{2}, m_{3}, m_{5}, \ldots$ in this order)

$$
\zeta(j):=\frac{(d+1)(d+2)}{2}-\frac{m_{1}\left(m_{1}+1\right)}{2}-\frac{m_{2}\left(m_{2}+1\right)}{2}-\frac{m_{3}\left(m_{3}+1\right)}{2}-\sum_{k=5}^{j+1} \frac{m_{k}\left(m_{k}+1\right)}{2} .
$$

As long as $\zeta(j) \geqslant\left(2 m_{4}-1\right)\left(m_{4}-1\right)+1$ holds, the reduction is possible. Note that $\zeta(s-1)=\operatorname{vdim} \mathcal{L}\left(d ; m_{1}, \ldots, m_{s}\right)+1+m_{4}\left(m_{4}+1\right) / 2$. If $\zeta(s-1)$ is greater or equal to $\left(2 m_{4}-1\right)\left(m_{4}-1\right)+1$, then all reductions are possible.

An immediate corollary useful for the proof of Theorem B is the following.

Corollary 3.4. Keeping the notation from Theorem 3.3, if the system in the theorem is $h^{1}$-regular, then the Castelnuovo-Mumford regularity of the ideal $I=\bigcap_{j=1}^{s} \mathfrak{m}_{p_{j}}^{m_{j}}$ is less than or equal to $d+1$.

Remark 3.5. Continuing Example 2.3, we observe that Theorem 3.2 implies that the system $\mathcal{L}(9 ; 4,4,4,3,3,3,3)$ is $h^{1}$-regular. With Theorem 3.3 we can show only that $\mathcal{L}(9 ; 4,4,4,3,3)$ is $h^{1}$-regular. In fact, from the proof of Theorem 3.3 we know that performing three 4 reductions and two 3 -reductions on $(1, \ldots, 10)$ is possible, even without performing them as in Example 2.3. This example shows that in some situations Theorem 3.2 is stronger than Theorem 3.3. However, Theorem 3.2 is simply not so useful in handling general situations.

\section{The containment results}

The following result of Harbourne and Huneke [6, Proposition 3.10] has motivated this section of the article.

TheOREM 4.1 (Harbourne and Huneke). Let $\mathcal{I}=\mathfrak{m}_{P_{1}} \cap \ldots \cap \mathfrak{m}_{P_{s}}$ be an ideal supported on $s$ general points in $\mathbb{P}^{2}$. Then

$$
\mathcal{I}^{(2 r)} \subset \mathcal{M}^{r} \mathcal{I}^{r}
$$

for all $r \geqslant 1$.

Our arguments in this section rely on the following fact modelled on a result by Harbourne and Huneke [6, Lemma 2.3].

Lemma 4.2. Let $\mathcal{I}$ be a homogeneous ideal in $(n+1)$ variables with 0-dimensional support. Assume that for some non-negative integers $r$ and $k$

$$
\alpha\left(\mathcal{I}^{(q)}\right) \geqslant r \cdot \operatorname{reg}(\mathcal{I})+k .
$$

Then

$$
\mathcal{I}^{(q)} \subset \mathcal{M}^{k} \cdot \mathcal{I}^{r}
$$


Proof. Note that since $\alpha\left(\mathcal{I}^{(q)}\right) \leqslant q \cdot \alpha(\mathcal{I})$, we get from (11) that $r \leqslant q$, which in particular shows the inclusion $\mathcal{I}^{(q)} \subset \mathcal{I}^{(r)}$.

For the Castelnuovo-Mumford regularity in our situation, we have by [5, Theorem 1.1]

$$
\operatorname{satdeg}\left(\mathcal{I}^{r}\right) \leqslant \operatorname{reg}\left(\mathcal{I}^{r}\right) \leqslant r \cdot \operatorname{reg}(\mathcal{I})
$$

so that

$$
\left(\mathcal{I}^{(r)}\right)_{t}=\left(\mathcal{I}^{r}\right)_{t} \quad \text { for } t \geqslant r \cdot \operatorname{reg}(\mathcal{I})
$$

Hence,

$$
\left(\mathcal{I}^{(q)}\right)_{t} \subset\left(\mathcal{I}^{r}\right)_{t} \quad \text { for } t \geqslant r \cdot \operatorname{reg}(\mathcal{I}) .
$$

Let $h_{1}, \ldots, h_{\ell}$ be minimal degree (that is, $\leqslant r \cdot t$ ) generators of $\mathcal{I}^{r}$. For $f \in\left(\mathcal{I}^{(q)}\right)_{t}$ we have $f=0$ if $t<r \cdot \operatorname{reg}(\mathcal{I})+k$ by the assumption (11). On the other hand, for $t \geqslant r \cdot \operatorname{reg}(\mathcal{I})+k$ by (12) there exist homogeneous polynomials $f_{1}, \ldots, f_{\ell}$ such that $f=\sum_{i=1}^{\ell} f_{i} \cdot h_{i}$. It follows that

$$
\operatorname{deg}\left(f_{i}\right) \geqslant \operatorname{deg}(f)-\operatorname{deg}\left(h_{i}\right) \geqslant k
$$

for all $i=1, \ldots, \ell$, which provides the desired result.

As an immediate consequence of the above lemma, we obtain the following useful criterion.

Corollary 4.3. Let $\mathcal{I}$ be a fat points ideal in $\mathbb{P}^{2}$. Assume that

$$
\alpha\left(\mathcal{I}^{(2 r)}\right) \geqslant r \cdot(\operatorname{reg}(\mathcal{I})+1) .
$$

Then

$$
\mathcal{I}^{(2 r)} \subset \mathcal{M}^{r} \mathcal{I}^{r}
$$

Before proceeding, it is convenient to introduce the following function

$$
\rho(m):= \begin{cases}0 & \text { if } m=1, \\ (3 m-1)(m-2) & \text { if } m \geqslant 2 .\end{cases}
$$

The crucial point in the proof of Theorem B is the following criterion, which follows from the $h^{1}$-regularity statement in Theorem 3.3.

TheOREM 4.4. Let $m_{1} \geqslant m_{2} \geqslant \ldots \geqslant m_{s}$ be positive integers and consider the fat points ideal $\mathcal{I}=\mathfrak{m}_{P_{1}}^{m_{1}} \cap \mathfrak{m}_{P_{2}}^{m_{2}} \cap \ldots \cap \mathfrak{m}_{P_{s}}^{m_{s}}$ of $s \geqslant 9$ general points. If there exists an integer $d$ such that

$$
d(d+3) \geqslant \sum_{i=1}^{s} m_{i}\left(m_{i}+1\right)+\rho\left(m_{4}\right)
$$

and

$$
d \geqslant m_{1}+m_{2}
$$

and

$$
d+2 \leqslant \max \left\{\frac{2}{\sqrt{s+1}} \sum_{i=1}^{s} m_{i}, m_{1}+m_{2}+m_{3}+m_{4}, 2 m_{1}\right\},
$$

then $\mathcal{I}^{(2 r)} \subset \mathcal{M}^{r} \mathcal{I}^{r}$ for all $r \geqslant 1$.

Proof. It follows from Theorem 3.3 that (13) and (14) imply that $\operatorname{reg}(\mathcal{I}) \leqslant d+1$, so that $\mathcal{I}$ is generated in degree $d+1$. In order to apply Corollary 4.3 we have to check that the inequality $\alpha\left(\mathcal{I}^{(2 r)}\right) \geqslant r(d+2)$ holds. 
By assumption (15) we need to consider three cases.

Assume first that

$$
d+2 \leqslant \frac{2}{\sqrt{s+1}} \sum_{i=1}^{s} m_{i} .
$$

A lower bound on Seshadri constants in $s \geqslant 9$ general points in $\mathbb{P}^{2}$, see for example $[\mathbf{9}$, Theorem $1(\mathrm{a})]$

$$
\varepsilon\left(\mathcal{O}_{\mathbb{P}^{2}}(1), P_{1}, \ldots, P_{s}\right) \geqslant \frac{1}{\sqrt{s+1}},
$$

combined with (16) implies that $\left(\mathcal{I}^{(2 r)}\right)_{r(d+2)}$ is empty so that $\alpha\left(\mathcal{I}^{(2 r)}\right)>r(d+2)$ in that case.

In the second case we assume

$$
d+2 \leqslant m_{1}+m_{2}+m_{3}+m_{4} .
$$

Then the standard Cremona transformation applied to the system $\mathcal{L}(r(d+2)-1$; $\left.2 m_{1}, \ldots, 2 m_{s}\right)$ gives

$$
\begin{aligned}
& \mathcal{L}\left(2 r\left(d+2-m_{1}-m_{2}-m_{3}-1\right)\right. \\
& \quad r\left(d+2-2 m_{2}-2 m_{3}\right)-1, r\left(d+2-2 m_{1}-2 m_{3}\right)-1, \\
& \left.\quad r\left(d+2-2 m_{1}-2 m_{2}\right)-1,2 m_{4}, \ldots, 2 m_{s}\right)
\end{aligned}
$$

which is obviously empty since its degree is less than the fourth multiplicity.

Finally, from the assumption $d+2 \leqslant 2 m_{1}$ it follows immediately that

$$
\left(\mathcal{I}^{(2 r)}\right)_{r(d+2)-1}=0
$$

and we are done.

REMARK 4.5. Computer experiments suggest that for nearly all sequences of multiplicities the assumptions of Theorem 4.4 are fulfilled. It took some time (and without computer aid it would not be so easy) to find some examples, where Theorem 4.4 does not prove the desired inclusion. Such sequences of multiplicities are for example

$$
\left(8^{9}, 1^{103}\right) ; \quad\left(9^{11}, 1^{80}\right) ; \quad\left(20^{12}, 2^{90}\right) ; \quad\left(30^{11}, 3^{130}\right) ; \quad\left(60^{11}, 5^{224}\right) ; \quad\left(130^{12}, 12^{101}\right),
$$

where the notation $a^{b}$ means that $a$ appears in the sequence $b$ times. All of these examples are of a similar nature, namely few points with high multiplicity and a long tail of low multiplicities. It would be interesting to find at least bounds on the initial degree of ideals associated to them in the spirit of the proof of Theorem 4.4.

In the following, we will frequently use the following purely numerical observation.

Lemma 4.6. Let $R, D \geqslant 0$ be real numbers. If $R^{2}-3 R \geqslant D$, then there exists an integer $d$ such that:

(a) $d(d+3) \geqslant D$; and

(b) $d+2 \leqslant R$.

Proof. Let $L_{0}:=(\sqrt{9+4 \cdot D}-3) / 2$ be the greater root of the equation $L(L+3)-D=0$. Obviously for any $d \geqslant L_{0}$ condition (a) in the lemma holds. In order to check condition (b), we show that there exists an integer $d$ in the interval $\left[L_{0}, R-2\right]$. This is immediate, once we show that the length of this interval is at least 1 , that is $R-2-L_{0} \geqslant 1$ holds. But this follows immediately from the assumption in the lemma. 
The proof of Theorem B will be split in two cases.

\subsection{Proof of Theorem B(a)}

We begin with the overview of the structure of the proof of part (a) of Theorem B. It follows from: Theorem 4.1 for $m_{1}=\ldots=m_{s}=1$; Proposition 4.7 for $m_{1} \geqslant 2$ and $m_{2}=\ldots=m_{s}=1$; and Theorem 4.9 for all other cases. It is convenient in the almost-homogeneous case we study here to change the notation a little bit and begin the numbering of points by 0 rather than 1 , the point $P_{0}$ being the point with distinguished multiplicity. This convention simplifies the notation below. We hope that this will cause no confusion and the reader will have no difficulties in modifying Theorem B(a) accordingly.

Proposition 4.7. Let $P_{0}, \ldots, P_{s}$ be general points on $\mathbb{P}^{2}$, with $s+1 \geqslant 9$. Let $\mathcal{I}=\mathfrak{m}_{P_{0}}^{m_{0}} \cap$ $\mathfrak{m}_{P_{1}} \cap \ldots \cap \mathfrak{m}_{P_{s}}, m_{0} \geqslant 2$. Then $\mathcal{I}^{(2 r)} \subset \mathcal{M}^{r} \mathcal{I}^{r}$ for all $r \geqslant 1$.

Proof. Assume that the regularity of $\mathcal{I}$ is $t$. The points being general impose independent conditions, so that we have

$$
\left(\begin{array}{l}
t \\
2
\end{array}\right)<s+\left(\begin{array}{c}
m_{0}+1 \\
2
\end{array}\right)
$$

We claim that $\alpha\left(\mathcal{I}^{(2 r)}\right) \geqslant r(t+1)$. Taking this for granted for the moment, the assertion follows from Lemma 4.2 .

Turning to the claim, the lower bound on Seshadri constants (17) implies that

$$
\alpha\left(\mathcal{I}^{(2 r)}\right) \geqslant \frac{2 r\left(m_{0}+s\right)}{\sqrt{s+2}} .
$$

Since the multiplicity of an element in $\mathcal{I}^{(2 r)}$ in the distinguished point is $2 r m_{0}$ we have additionally

$$
\alpha\left(\mathcal{I}^{(2 r)}\right) \geqslant 2 r m_{0}
$$

Thus, dividing by $r$, it suffices to prove that the following

$$
2 \frac{m_{0}+s}{\sqrt{s+2}} \geqslant t+1 \quad \text { or } 2 m_{0} \geqslant t+1
$$

holds for all $t$ satisfying (18).

Assume that (19) does not hold and plug

$$
t=2 \frac{m_{0}+s}{\sqrt{s+2}}-1
$$

into (18). After a small computation we obtain the inequality

$$
\frac{4 m_{0}^{2}+8 m_{0} s+4 s^{2}}{s+2}+2 \leqslant 2 s+m_{0}^{2}+m_{0}+\frac{6 m_{0}}{\sqrt{s+2}}+\frac{6 s}{\sqrt{s+2}} .
$$

Let us assume that $s \geqslant 34$, so that in particular $6 / \sqrt{s+2} \leqslant 1$. Then

$$
\frac{4 m_{0}^{2}+8 m_{0} s+4 s^{2}}{s+2} \leqslant 3 s+m_{0}^{2}+2 m_{0}
$$

hence

$$
2 m_{0}^{2}+6 m_{0} s+s^{2} \leqslant 6 s+s m_{0}^{2}+4 m_{0} .
$$

But for $m_{0}^{2} \leqslant s$ we have

$$
2 m_{0}^{2}>4 m_{0}, \quad 6 m_{0} s>6 s \text { and } s^{2} \geqslant s m_{0}^{2},
$$

which gives a contradiction. 
Now we do the same for $t=2 m_{0}-1$ obtaining

$$
3 m_{0}^{2}-7 m_{0}+2 \leqslant 2 s
$$

and observe that for $s \leqslant m_{0}^{2}$ and $m_{0} \geqslant 7$ this is absurd.

Thus, we are left with a finite number of pairs $\left(m_{0}, s\right)$ to check, namely $m_{0}^{2} \leqslant s \leqslant 33$ and $s \leqslant m_{0}^{2} \leqslant 36$. In each case we directly compute maximal possible $t$ satisfying (18) and check that it fits to (19).

In order to prove Theorem 4.9 below, we need first the following lemma. We abbreviate $\Sigma=s m+m_{0}$ and $Q=s m^{2}+m_{0}^{2}$.

LEMMA 4.8. Let $m_{0} \geqslant 1, m \geqslant 2$ and $s \geqslant 8$ be integers:

(a) if $m_{0} \geqslant \Sigma / \sqrt{s+2}$, then $4 m_{0}^{2} \geqslant Q+\Sigma+3 m^{2}+6 m_{0}$;

(b) if $m_{0} \leqslant \Sigma / \sqrt{s+2}$, then $(4 /(s+2)) \Sigma^{2} \geqslant m_{0}^{2}+(s+3) m^{2}+3 \Sigma$.

Proof. Part (a) An elementary computation shows that the assumption in Part (a) together with the following inequality

$$
3\left(m_{0}+s m\right)^{2} \geqslant(s+2)(s+3) m^{2}+7(s+2) m_{0}+s(s+2) m
$$

implies the assertion. So it is enough to show (20). It follows, since for $s \geqslant 8$ and $m \geqslant 2$ the following inequalities are satisfied:

$$
\begin{gathered}
2 s^{2} m^{2} \geqslant(s+2)(s+3) m^{2}, \\
s^{2} m^{2} \geqslant s(s+2) m, \\
3 m_{0}^{2}+6 s m m_{0} \geqslant 7(s+2) m_{0} .
\end{gathered}
$$

Adding them gives (20).

Part (b) Adding

$$
\Sigma^{2} \geqslant(s+2) m_{0}^{2},
$$

which follows from the assumption in this part, to the following inequality

$$
3\left(m_{0}+s m\right)^{2} \geqslant(s+2)(s+3) m^{2}+3(s+2)\left(m_{0}+s m\right)
$$

one obtains the inequality claimed in part (b). So it suffices to prove (21). For all $s \geqslant 8$ and $m \geqslant 2$ we have

$$
3 m_{0}+6 s m \geqslant 3 s+6,
$$

so that it is enough to check that

$$
3 s^{2} m \geqslant(s+2)(s+3) m+3(s+2) s .
$$

For $s \geqslant 8$ we have

$$
2 s^{2} \geqslant(s+2)(s+3)
$$

so $(22)$ reduces to

$$
m s^{2} \geqslant 3 s^{2}+6 s .
$$

This inequality is satisfied for all $m \geqslant 4$.

For $m=2$ or $m=3$ we show (22) slightly differently. Namely, we bound the summands on the right-hand side in the following way

$$
\frac{5}{4} s^{2} \geqslant(s+2)(s+3) \text { and } \frac{7}{4} s^{2} m \geqslant 3(s+2),
$$

which holds for all $s \geqslant 22$.

In all remaining, finitely many cases, that is, $m=2$ or $m=3$ and $8 \leqslant s \leqslant 21$, we check the inequality in part (b) directly by hand.

We are now in the position to justify part (a) of Theorem B. 
Theorem 4.9. Let $m_{0} \geqslant 1$ and $m \geqslant 2$ be integers. Consider the ideal $\mathcal{I}=\mathfrak{m}_{P_{0}}^{m_{0}} \cap \mathfrak{m}_{P_{1}}^{m} \cap \ldots \cap$ $\mathfrak{m}_{P_{s}}^{m}$ of $s+1 \geqslant 9$ general (fat) points. Then $\mathcal{I}^{(2 r)} \subset \mathcal{M}^{r} \mathcal{I}^{r}$ for all $r \geqslant 1$.

Proof. Let $D=m_{0}\left(m_{0}+1\right)+s \cdot m(m+1)+\rho(m)$.

If $m_{0} \geqslant\left(m_{0}+s m\right) / \sqrt{s+2}$ we put $R=2 m_{0}$. Then part (a) of Lemma 4.6 implies

$$
R^{2}-3 R \geqslant D
$$

in this case.

If $m_{0} \leqslant\left(m_{0}+s m\right) / \sqrt{s+2}$ we put $R=2\left(m_{0}+s m\right) / \sqrt{s+2}$. Then part (b) in Lemma 4.6 implies again (24) in this case.

Thus, (24) is always satisfied and Lemma 4.6 implies that there exists $d$ satisfying (13) and (15). If this $d$ satisfies also (14), then we are done.

Otherwise we work with a new $d^{\prime}:=m_{1}+m_{2}>d$. This $d^{\prime}$ satisfies obviously (14) and also (13) and (15) as $d^{\prime}+2 \leqslant m_{1}+m_{2}+m_{3}+m_{4}$. Thus, Theorem 4.4 gives the assertion.

\subsection{Proof of Theorem $B(b)$}

We work under the assumption

$$
m_{s} \geqslant \frac{m_{1}}{2}
$$

In the following we will use again the following notation:

$$
\Sigma=m_{1}+\ldots+m_{s} \text { and } Q=m_{1}^{2}+\ldots+m_{s}^{2} .
$$

Aiming for Theorem 4.11 we prove first the following purely numerical lemma.

LEMmA 4.10. With the above notation and assuming $s \geqslant 9$ we have

$$
\frac{4}{s+1} \Sigma^{2} \geqslant Q+\left(\frac{6}{\sqrt{s+1}}+1\right) \Sigma+\varrho\left(m_{4}\right)
$$

except for $m_{1}=2, m_{2}=\ldots=m_{s}=1$.

Proof. We relax the assumption that $m_{i}$ are integers (but we keep the monotonicity assumption $\left.m_{1} \geqslant m_{2} \ldots \geqslant m_{s} \geqslant m_{1} / 2\right)$. Working with fixed $m_{1}, m_{2}, m_{3}, m_{4}$ and $\Sigma$ we adjust all other $m_{i}$ so that the right-hand side of (25) becomes maximal possible. Observe that if we subtract from an $m_{k}$ a positive number $a$ and we add this number to a bigger $m_{j}$ with $j>4, \Sigma$ will remain the same and $Q$ will become bigger. We proceed in this way to obtain the sequence of $m_{i}$ satisfying the assumed conditions and maximizing the right-hand side. The sequence becomes

$$
m_{1}, m_{2}, m_{3}, m_{4}=m_{5}=\ldots=m_{t}, m_{t+1}, \frac{m_{1}}{2}, \ldots, \frac{m_{1}}{2} \quad \text { with } t \geqslant 4
$$

where $m_{1} / 2$ stands on positions $t+2, \ldots, s$ and we have $m_{1} / 2 \leqslant m_{t+1} \leqslant m_{4}$.

Now, the idea is to reduce inequality (25) to inequality (26) below, which is easier to prove later. To alleviate the notation we put $x:=m_{1}$ and $y:=m_{4}$.

First we bound the left-hand side of the inequality (25) from below:

$$
\frac{4}{s+1} \Sigma^{2} \geqslant \frac{4}{s+1}\left(x+(t-1) y+(s-t) \frac{x}{2}\right)^{2}=\frac{4}{s+1}\left((t-1) y+(s-t+2) \frac{x}{2}\right)^{2} .
$$


The right-hand side of the inequality (25) can in turn be bounded from above replacing $m_{2}$ and $m_{3}$ by $x, m_{t+1}$ by $y$ and finally $\varrho(y)$ by $3 y^{2}$ as follows:

$$
\begin{aligned}
Q+ & \left(\frac{6}{\sqrt{s+1}}+1\right) \Sigma+\varrho(y) \leqslant\left(3 x^{2}+(t-2) y^{2}+(s-t-1) \frac{x^{2}}{4}\right) \\
& +\left(\frac{6}{\sqrt{s+1}}+1\right)\left(3 x+(t-2) y+(s-t-1) \frac{x}{2}\right)+3 y^{2} \\
= & \left((t+1) y^{2}+(s-t+11) \frac{x^{2}}{4}\right)+\left(\frac{6}{\sqrt{s+1}}+1\right)\left((t-2) y+(s-t+5) \frac{x}{2}\right) .
\end{aligned}
$$

Thus, (25) will follow from

$$
\begin{aligned}
4\left((t-1) y+(s-t+2) \frac{x}{2}\right)^{2} \geqslant & (s+1)\left((t+1) y^{2}+(s-t+11) \frac{x^{2}}{4}\right) \\
& +\left(\frac{6}{\sqrt{s+1}}+1\right)\left((t-2) y+(s-t+5) \frac{x}{2}\right) .
\end{aligned}
$$

Bounding $6 / \sqrt{s+1}$ from above by 2 , multiplying by 4 and bringing everything on one side, we get a new inequality which implies (26) of course:

$$
\begin{aligned}
& \left(3 x^{2}-6 x\right) s^{2}+\left(16 x y-7 x^{2}-4 y^{2}+6 x-12 y\right) s t+4\left(x^{2}+4 y^{2}-4 x y\right) t^{2} \\
& \quad+4\left(x^{2}-y^{2}-4 x y-9 x+6 y\right) s+3\left(16 x y-5 x^{2}-12 y^{2}+2 x-4 y\right) t \\
& \quad+5 x^{2}+12 y^{2}-32 x y+24 y-30 x \geqslant 0
\end{aligned}
$$

with constraints

$$
x \geqslant y, \quad 2 y \geqslant x, \quad s \geqslant 9, \quad t \geqslant 4 \text { and } s \geqslant t .
$$

Now, we prove inequality (27) under the additional assumption $x \geqslant 4$. It can be easily checked that the coefficients at $t^{2}$ and $s t$ are always positive under conditions (28).

Similarly we have positivity for slightly modified (by the underlined terms) coefficients at $s$, $t$ and the constant term

$$
\begin{aligned}
\underline{19 x^{2}}+4\left(x^{2}-y^{2}-4 x y-9 x+6 y\right) & \geqslant 0, \\
\underline{\frac{9}{2} x^{2}}+3\left(16 x y-5 x^{2}-12 y^{2}+2 x-4 y\right) & \geqslant 0, \\
\frac{33}{2} x^{2}+5 x^{2}+12 y^{2}-32 x y+24 y-30 x & \geqslant 0 .
\end{aligned}
$$

The proof will be finished, once we show that the inequality

$$
6 x^{2} s^{2} \geqslant 12 x s^{2}+38 x^{2} s+9 x^{2} t+33 x^{2}
$$

is satisfied under conditions (28). Using the estimate $t \leqslant s$ and dividing by $x$ this is reduced to

$$
6 x s^{2} \geqslant 12 s^{2}+(38 x s+9 x s+33 x) .
$$

For $s \geqslant 17$, the term $3 x s^{2}$ bounds both summands on the right-hand side in (30) for all $x \geqslant 4$. Similarly, for $x \geqslant 34$ we can bound both summands in the following manner

$$
0.36 x s^{2} \geqslant 12 s^{2} \text { and }(6-0.36) x s^{2} \geqslant 38 x s+9 x s+33 x
$$

for all $s \geqslant 9$.

Thus, we are left with finitely many cases, namely

$$
9 \leqslant s \leqslant 16 \text { and } 4 \leqslant x \leqslant 33
$$

for which we check (27) directly by some dull computations omitted here. 
It remains to check (25) with cases $x=3$ and $x=2$ which we leave to a motivated enough reader. Note that lemma is not true in the case $x=2$ and $m_{2}=\ldots=m_{s}=1$ (which is covered by Proposition 4.7).

We are now in the position to finish the proof of part (b) of Theorem B.

TheOREM 4.11. Let $m_{1} \geqslant m_{2} \geqslant \ldots \geqslant m_{s} \geqslant m_{1} / 2$ be positive integers. We consider the ideal $\mathcal{I}=\mathfrak{m}_{P_{1}}^{m_{1}} \cap \mathfrak{m}_{P_{2}}^{m_{2}} \cap \ldots \cap \mathfrak{m}_{P_{s}}^{m_{s}}$ of $s \geqslant 9$ general (fat) points. Then $\mathcal{I}^{(2 r)} \subset \mathcal{M}^{r} \mathcal{I}^{r}$ for all $r \geqslant 1$.

Proof. The case $m_{1}=\ldots=m_{s}=1$ is covered by Theorem 4.1.

The case $m_{1}=2$ and $m_{2}=\ldots m_{s}$ follows from Proposition 4.7 .

In the remaining cases the inequality (25) in Lemma 4.10 is equivalent to inequality

$$
R^{2}-3 R \geqslant D
$$

if we define

$$
D=\sum m_{i}\left(m_{i}+1\right)+\varrho\left(m_{4}\right) \quad \text { and } \quad R=\frac{2}{\sqrt{s+1}} \sum m_{i}
$$

By Lemma 4.6 we know then that there exists an integer $d$ satisfying (13) and (15). If $d \geqslant m_{1}+m_{2}$ we are done by Theorem 4.4. Otherwise, exactly as in the proof of Theorem 4.9, we take $d^{\prime}=m_{1}+m_{2}$ and we observe that since $d^{\prime}+2 \leqslant m_{1}+m_{2}+m_{3}+m_{4}$, the integer $d^{\prime}$ satisfies inequalities (13) and (15); so that Theorem 4.4 ends the proof.

\section{References}

1. C. Ciliberto, 'Geometric aspects of polynomial interpolation in more variables and of Waring's problem', European Congress of Mathematics, Vol. I (Barcelona, 2000), Progress in Mathematics 201 (Birkhäuser, Basel, 2001) 289-316.

2. M. Dumnicki, 'Symbolic powers of ideals of generic points in $\mathbb{P}^{3}$ ', J. Pure Appl. Algebra 216 (2012) 1410-1417.

3. M. DumNiCKI and W. JARNiCKI, 'New effective bounds on the dimension of a linear system in $\mathbb{P}^{2}$, J. Symbolic Comp. 42 (2007) 621-635.

4. L. Ein, R. LAzARSFELD and K. SMith, 'Uniform bounds and symbolic powers on smooth varieties', Invent. Math. 144 (2001) 241-252.

5. A. Geramita, A. Gimigliano and Y. Pitteloud, 'Graded Betti numbers of some embedded rational n-folds', Math. Ann. 301 (1995) 363-380.

6. B. Harbourne and C. Huneke, 'Are symbolic powers highly evolved?', J. Ramanujan Math. Soc. 28 (2013) 311-330.

7. M. Hochster and C. Huneke, 'Comparison of symbolic and ordinary powers of ideals', Invent. Math. 147 (2002) 349-369.

8. S. Sullivant, 'Combinatorial symbolic powers', J. Algebra 319 (2008) 115-142.

9. G. Xu, 'Curves in $\mathbb{P}^{2}$ and symplectic packings', Math. Ann. 299 (1994) 609-613.

M. Dumnicki

Institute of Mathematics

Jagiellonian University

Łojasiewicza 6, PL-30-348 Kraków

Poland

marcin.dumnicki@im.uj.edu.pl

H. Tutaj-Gasińska

Institute of Mathematics

Jagiellonian University

Łojasiewicza 6, PL-30-348 Kraków

Poland

halszka.tutaj@im.uj.edu.pl

\section{T. Szemberg}

Institute of Mathematics

Pedagogical University of Cracow

Podchorazych 2, PL-30-084 Cracow

Poland

szemberg@up.krakow.pl 